\title{
Rechtsgeschichte
}

http://www.rg-rechtsgeschichte.de/rg13

Zitiervorschlag: Rechtsgeschichte Rg 13 (2008)

$\operatorname{Rg} \mathbf{3}_{2008}$

$211-213$

http://dx.doi.org/10.12946/rg13/211-213

\section{Petra Gehring}

\section{Vielgelesenes Vorwort}

Gumbrecht zur Begriffsgeschichte 
schenrechtsdebatte und über derzeit übliche Begründungsformen diskutiert er extensiv alle einschlägigen Einlassungen Luhmanns, um schließlich Vorbehalte geltend zu machen, die dessen gesamte Perspektive in Frage stellen.

In nicht ganz deutlicher Rangordnung unterstellt er der Systemtheorie einerseits methodologisch den Anspruch einer »naturwissenschaftlichen Verankerung « (348), wobei das Theorem der funktionalen Differenzierung von Gesellschaft nicht mehr sein könne als der Versuch, das »evolutive Prinzip als eine Gesetzmäßigkeit " (349) zu behaupten. Ganz grundlegend hält er aber andererseits die systemtheoretische Perspektive auf den Menschen und seine Rechte für verfehlt: Luhmanns Soziologisierung rede einer »Unterwerfung durch die Gesellschaft " (305), sogar einer »Entwürdigung des Einzelnen " als Folge der Theorie (35I) das Wort, da ihr ein »menschenentwürdigender Funktionalismus zugrunde $(3)$ liege.

Die erhobenen Einwände werden allerdings zunehmend fragwürdig, wenn Noll diese Pro-

\section{Vielgelesenes Vorwort}

Gumbrecht zur Begriffsgeschichte*

Er sei »von Begriffsgeschichten umstellt«, so eröffnet der in Stanford lehrende Literaturwissenschaftler Hans Ulrich Gumbrecht das lange Vorwort eines 2006 erschienenen Bandes, in welchem er sechs begriffgeschichtliche Artikel in unveränderter Form wieder abdruckt, die von ihm in den Jahren 1978 bis $200 \mathrm{I}$ erschienen sind - zum größten Teil in Nachschlagewerken, die wir bestens kennen. Wer das Buch erwarb, hatte also vor allem ein Vorwort gekauft. Aller- bleme forsch auf die dieser Theorie angeblich zugrunde liegende »säkularisiert-materialistische Geisteshaltung « (357, vgl. 43I) zurückführt und in ambitionierten Exkursen versucht, eine grundlegende metaphysische Alternative zu formulieren, als deren Ergebnis sich ein völlig neuer Begriff der Zeit und ein neues Verständnis von Geist und Materie abzeichnen und mit dessen Hilfe sich »aus der Natur des Seins « (435) ein »Menschen- und Erdrechtsschema « (450) ableiten lassen soll. Mit diesen Überlegungen, scheint es, hat aber Noll den Orbit der Luhmannschen Rechtssoziologie, der es übrigens um nichts weniger als eine »Begründung der Menschenrechte" ging, längst verlassen, und die verschmähte Soziologisierung erscheint im Vergleich wie eine attraktive Gegenoperation, deren Konsequenzen für die Fragen nach Geltung und Begründung, wie sie Menke/Pollmann mustergültig exponieren, allerdings nicht leicht abzusehen ist. Das letzte Wort über die Soziologie der Menschenrechte ist somit noch nicht gesprochen.

Martin Saar

dings starker Stoff: Gumbrecht verabschiedet die Begriffsgeschichte!

"Pyramiden des Geistes" heißt das Vorwort, welches »den schnellen Aufstieg, die unsichtbaren Dimensionen und das plötzliche $\mathrm{Ab}$ ebben der begriffsgeschichtlichen Bewegung " bespricht. Gumbrecht legt das Thema autobiografisch an. Seinerzeit verstand er es als Ehre, seine Zeit in die mühsame Textgattung begriffsgeschichtlicher Prosa zu stecken, er nutzt die

\footnotetext{
* Hans Ulrich Gumbrecht,

Dimensionen und Grenzen der

Begriffsgeschichte. München:

Wilhelm Fink Verlag 2006, 260 S.,

ISBN 978-3-7705-3694-8
} 
entstandenen umfangreichen Nachschlagewerke nach wie vor gern und reichlich. Und er bedauert seine amerikanischen Kollegen, denn Vergleichbares gibt es in englischer Sprache nicht. Gumbrecht fühlt sich in den Aufstieg des begriffsgeschichtlichen Paradigmas »hineingeboren « (9). Bis »vor kurzem « sei ihm die Tatsache entgangen, dass sich dieser Aufstieg »ähnlich ereignishaft, ja fast plötzlich vollzogen hatte wie nun am Ende des 20. Jahrhunderts die Versteinerung der lebhaften begriffsgeschichtlichen Arbeit in ein Monument der Vergangenheit « (9). Daher der Seufzer: "Was sollen wir heute anfangen mit den in Pyramidenferne gerückten Sedimenten unserer intellektuellen Jugend? « (9)

Ja so ist das, könnte man sagen: Irgendwann kommen auch strukturell unabschließbare Projekte an ein Ende, vor allem, wenn sie ans Abarbeiten des Alphabets gebunden sind. Und älter wird man auch. Aber Gumbrecht meint natürlich etwas anderes: Die Petrifikation kommt von innen. In den geistigen Pyramiden ist kein Leben mehr. Bei der Sisyphosarbeit ist das Herz dessen versteinert, der die Steine rollt.

Die Diagnose wird konkretisiert durch eine geraffte Rekonstruktion der Entstehungsgeschichten der beiden seit den I970er Jahren epochemachenden begriffsgeschichtlichen Projekte »Historisches Wörterbuch der Philosophie« (Ritter, Gründer, Gabriel) und » Geschichtliche Grundbegriffe " (Brunner, Conze, Koselleck) sowie des nach der Wende in Angriff genommenen »Wörterbuchs Ästhetischer Grundbegriffe « (Barck u. a.). Jeweils - so der Tenor hatte Begriffsgeschichte letztlich kompensatorische Funktion. Und da sind verborgene "Sonderdimensionen " (28): eine unbestimmte Beziehung zur Sachgeschichte, die als Sicherheitsabstand gegenüber der »Weltreferenz der Sprache " (27) diene, ein im Ganzen unfestgelegter Erkenntnis- anspruch sowie in der Konzentration auf Begriffe ein nachkriegstypisches Latenthalten der "nationalen Geschichte - und damit der eigenen NS-Vergangenheit ( $3 \circ \mathrm{f}$.). Mit letzterem Punkt folgt Gumbrecht dem Literaturwissenschaftler Anselm Haverkamp, einem Kritiker der Begriffsgeschichte als politisch motivierte »Begriffsbereinigungsgeschichte ${ }^{\mathrm{I}}$ und Streiter für die Metaphorologie des späten Blumenberg.

Gumbrecht erweitert, warum er nun die Sachen packt und wohin die Reise gehen soll, zur Zeitdiagnose. Was einst den Erfolg der Begriffsgeschichte bedingte, sei der Grund, warum sie heute enttäusche. Zwischen Realismen und Konstruktivismen seien in der Postmoderne Entscheidungen in Sachen Weltverhältnis fällig. Begriffsgeschichte erscheine nurmehr als Ideengeschichte im schlechten Sinn. Philosophische. Was die begriffsgeschichtliche Praxis daher »abzulösen oder mindestens zu ergänzen « hätte, sei die Metaphorologie - Metaphorologie als Möglichkeit, sich auf jene Wirklichkeiten einzulassen, »welche in Sprache gegenwärtig, aber nicht begrifflich werden können ", auf jenes "Sein, das nicht vollends zur Sprache wird, weil es nie ganz zu verstehen ist « $(36)$.

Ist diese heideggerianisch anmutende Wendung überzeugend? Und trifft das ganze Vorwort tatsächlich den Nerv eines Problems? Jedenfalls waren Gumbrechts »Pyramiden « eine Provokation. Die Reaktionen fallen allerdings unterschiedlich aus und eine Debatte ist aus dem Anstoß nicht geworden. Stephan Schlak beschmunzelt eine unverkennbare Sentimentalität des Textes, sieht aber mit der Sehnsucht nach Präsenz und Wirklichkeit »eine akademische Grundstimmung des Tages getroffen ${ }^{2}{ }^{2}$ Helmuth Mayer ist skeptisch, was die Haverkamp-Adaptionen angeht, und stößt lieber auf die gegenwartsdiagnostische Hintergrundthese durch: Lässt die Post-

I Anselm Haverkamp, Metaphorologie zweiten Grades, in: DERS., Metapher. Die Ästhetik in der Rhetorik, München 2007, I45I63, I 59.

2 Stephan Schlak, Der Sound der Sentimentalität, in: Süddeutsche

Zeitung vom 2.I.2007. 
moderne tatsächlich den Stern der Begriffsgeschichte sinken? Er hält diese Frage für offen. ${ }^{3}$ Helmut Hühn moniert - neben systematischen Ungereimtheiten - Schräglagen der Gumbrechtschen Rekonstruktion der Entstehungsgeschichte des Historischen Wörterbuchs der Philosophie. Hühn empfiehlt den Lesern nicht das »Pyramiden «-Vorwort, sondern die begriffsgeschichtlichen Kapitel des Buchs. ${ }^{4}$ Zum förmlichen Schlagabtausch kommt es in der "Zeitschrift für Ideengeschichte «: Nachdem Carsten Dutt insbesondere Gumbrechts Epochen-Argument kritisiert - ist die Postmoderne wirklich ein neues »Chronotop «, mit welchem sich Begriffsgeschichte erübrigt? -, repliziert Gumbrecht sinngemäß, man müsse eben in den I970er und I980er Jahren dabei gewesen sein, um zu verstehen, was er meine. Und ad personam Dutt: Man habe es womöglich mit einem generationentypischen Temperamentsunterschied zu tun. ${ }^{5}$

Hat nun also eine begriffsgeschichtlich ernüchterte, dafür metaphorologische Ära begonnen? Oder schüttelt die begriffsgeschichtliche community angesichts vielen Pauschalisierungen und der laxen Verweise auf Haverkamp zu Recht nur den Kopf? Erstens könnte beides stimmen und zweitens hat Gumbrechts Vorwort ein Problem. Seine subjektive Kamera steht anderswo: Er kennt das Diskursklima nicht wirklich, von dem er spricht. Jedenfalls ihm ist ein Wandel entgangen, der die Begriffsgeschichte der I $990 e r$ Jahre betrifft. Längst schon sind ja die begriffsgeschichtlichen Großprojekte und ebenso die Metaphorologie zu Werkstätten geworden für die (inzwischen auch nicht mehr ganz junge) Generation derjenigen, die gar nicht mit der Gumbrechtschen Alternative Ritter, Gadamer oder Blumenberg sozialisiert sind, sondern epistemologisch denken - mit Luhmann arbeiten und mit der Diskursanalyse Foucaults. Für die
Begriffsgeschichte Koselleckscher Prägung moniert der Historiker Stefan Jordan, Gumbrecht sei auf dem Diskussionsstand der I990er Jahre stehengeblieben. ${ }^{6}$ Man kann aber auch die letzten Bände des »Historischen Wörterbuchs der Philosophie« unter diesem Aspekt zur Hand nehmen. Dort findet sich eine Fülle von methodologisch durchaus entschiedenen, an einer Epistemologie von Diskursen und auch an Blumenberg geschulten Beiträgen.

Gumbrechts Diagnose fixiert sich auf die frühen Programmatiken, die er zitiert. Ob deren Zurückhaltung in der Frage einer methodologischen Selbstfestlegung andere als pragmatische Gründe hatte, mag man diskutieren. Jedenfalls aber wurde jene »Bewegung " der Begriffsgeschichte nicht eingesperrt, und dem Einsickern des Neuen stand eben deshalb nichts im Wege. Auf diese Weise sind namentlich die die beiden Großprojekte »Ritter « und »Koselleck « über 40 Jahre hinweg ungemein veränderungsfreudig geblieben. Es ging fröhlich positivistisch zu und das Paradigma Begriffsgeschichte schärfte sich zusehends - ohne programmatisches Pathos. Und ohne übrigens im Mindesten einen Gegensatz zur historischen Metaphernforschung aufmachen zu müssen oder zu anderen Formen der Metaphernanalyse. Im Gegenteil: Auch die Lust auf Metaphern gedeiht bestens auf begriffsgeschichtlichem Untergrund.

Zeitgeist einzuschätzen ist schwierig. Gern hält man das für eine »Bewegung «, von dem man selbst erfasst war und übersieht, was dann danach wiederum andere bewegt hat und bewegt. Mag also sein, dass das Pyramidenproblem so groß gar nicht ist - und das Gumbrechtsche »Umstelltsein « von Begriffsgeschichten sich einfach beheben ließe, indem er seine Bücherregale umräumt.

Petra Gehring
3 Helmut Mayer, Der Stern der Begriffsgeschichte, in: Frankfurter Allgemeine Zeitung vom 26.3.2007.

4 Helmut Hühn, »Gut gebrüllt, Löwe? "Hans Ulrich Gumbrecht lässt von der Begriffsgeschichte $a b$, in: IASLonline [I 8.1 2.2007].

5 Carsten Dutt, Postmoderne $\mathrm{Zu}$ kunftsmüdigkeit. Hans Ulrich Gumbrecht verabschiedet die Begriffsgeschichte, in: Zeitschrift für
Ideengeschichte [ZIG] I/I (2007) I I8-I22; Hans UlRICH GUMBRECHT, (Un)dankbare Generationen. Eine Replik auf Carsten Dutt, in: ZIG I/3 (2007), I 22I24; Carsten Dutt, Keine Frage des Alters. Eine Duplik, in: ZIG I/3 (2007) I 25-I 27.

6 Stefan Jordan, Rezension "Hans Ulrich Gumbrecht: Dimensionen und Grenzen der Begriffsgeschichte«, in: Zeitschrift für Geschichts- wissenschaft 55 (2007) 457 f., hier 458 . 